\title{
Application of Drainage Position Ventilation and Real- Time Bedside Monitoring in Mechanical Ventilation of Patients Infected with nCov-19
}

\author{
Zhongping Cao* \\ Department of Anesthesia, The Special Characteristic Medicine Center of Chinese People Armed Police Force, Tianjin 300162, China \\ ${ }^{\star}$ Corresponding author: Zhongping Cao, Department of Anesthesia, The Special Characteristic Medicine Center of Chinese People Armed Police Force, Tianjin 300162, China
}

Received: November 18, 2020; Accepted: December 28, 2020; Published: December 31, 2020

\begin{abstract}
At present, the new coronavirus has spread to more than 200 countries and regions around the world. Up to now, no specific antiviral drugs are proved effective in defeating the new coronavirus, some measures, such as postural drainage ventilation, real-time bedside pulmonary ultrasound and chest electrical impedance monitoring may provide some new ideas for mechanical ventilation patients infected with new coronavirus.
\end{abstract}

Keywords: New coronavirus, ARDS, Mechanical ventilation, Bioelectrical impedance tomography, Pulmonary ultrasound

\section{Etiology and Pathogenesis}

The novel coronavirus (2019-nCoV) belongs to the beta genus of coronavirus, the $\mathrm{S}$ protein of the new coronavirus binds to the angiotensin-converting enzyme 2 (ACE2) receptor of human alveolar type II epithelial cells, and then enters into the cell to replicate and spread through respiratory droplets and contact [1].

\section{Clinical Manifestation}

Fever, dry cough and fatigue are the main symptoms of the people infected with novel coronavirus. Critically ill patients usually have dyspnea and (or) hypoxemia one week after the onset of the disease. Some patients can rapidly progress to acute respiratory distress syndrome, septic shock, uncorrectable metabolic acidosis, coagulation dysfunction and multiple organ failure [1].

\section{Chest Imaging}

Chest radiographs showed multiple small patch shadows and interstitial changes in the lungs, especially in the lateral pulmonary zone in the early stage of the patients infected with new coronavirus. Then it developed into multiple ground glass shadows and infiltration shadows in both lungs, and in severe cases, lung consolidation could occur [1-3].

\section{Pulmonary Pathophysiology}

Lung pathology showed focal hemorrhage and necrosis, marked proliferation of the type II alveolar epithelial cells in the lung tissue. Serous, fibrin exudates, and hyaline membrane formation were seen in the alveolar cavity; it could also be observed that the alveolar septal vascular congestion and edema, and some alveolar exudates organization and pulmonary interstitial fibrosis. Part of the bronchial mucosa epithelium was shed; mucus and mucus emboli could be seen in the bronchial lumen. A small number of alveoli were over-inflated, the alveolar septum was broken or the cysts were formed [4].

Thus, critically ill patients infected with new coronavirus may present abnormal pathophysiological changes such as obstructive ventilation disorder, lung gas exchange disorder, imbalanced ventilation blood flow ratio, and increased shunt.

\section{Antiviral Therapy}

During the emergency clinical trial of antiviral drugs, a number of randomized, double-blind, antiviral-placebo controlled studies have been carried out, but no antiviral drugs proved effective in treating the new coronavirus infection.

\section{Mechanical Ventilation}

Early and appropriate invasive mechanical ventilation is an important treatment for critically ill patients. In general, when $\mathrm{PaO}_{2} /$ $\mathrm{FiO}_{2}$ is less than $150 \mathrm{mmHg}$, the effect of high flow oxygen therapy or noninvasive ventilation is not good, endotracheal intubation should be considered in time for invasive mechanical ventilation in severe and critical ill cases [2]. The strategies of lung protective mechanical ventilation and lung recruitment are implemented. If there is no contraindication, it is suggested to implement prone position ventilation at the same time. Prone position ventilation can improve oxygenation in patients with ARDS by increasing functional residual volume, improving ventilation/blood flow ratio $(\mathrm{V} / \mathrm{Q})$, reducing shunt (Qs/Qt), improving diaphragmatic movement and promoting secretion excretion. In the airway management, posture drainage and sputum suction by bronchoscope should be adopted to promote the sputum drainage and lung rehabilitation [2]. 


\section{Lung Protective Mechanical Ventilation Strategy}

The individualized strategy of mechanical ventilation is to adopt the most suitable methods or parameters in ventilation mode, lung recruitment, tidal volume, PEEP and mechanical ventilation posture for patients according to their different pathophysiological conditions, so as to achieve the best treatment effect. At present, low tidal volume, high PEEP, lung recruitment and prone position ventilation are widely used in patients infected with new coronavirus. ${ }^{2}$ The characteristics of severe new coronavirus cases, such as inflammatory serous and fibrin exudate, exudate organization, pulmonary fibrosis, alveolar septum destruction, atelectasis and pulmonary bullae, coexist in the patients' lung [4]. Large tidal volume is not suitable for patients infected with new coronavirus due to the potential mechanical ventilation lung injury [2]. The selection of PEEP should be guided by the best pulmonary mechanics, the reduction of pulmonary shunt, the improvement of oxygenation and the function of stable circulation, while the effect of pulmonary recruitment should be examined by CT, MRI, bioelectrical impedance tomography (EIT) and ultrasound imaging. In the process of lung recruitment, there is the possibility of lung over inflation and the original pulmonary injury aggravation, and the effect on the hemodynamics should be concerned at the same time. The optimal method, opportunity and parameters of lung recruitment have not been determined, but it is necessary to judge the potential of pulmonary reinflation under real-time bedside EIT and ultrasound pulmonary monitoring.

\section{The Advantage of Real Time Bedside Monitoring of EIT and Ultrasound}

The goal-oriented mechanical ventilation is to adjust the mechanical ventilation strategy in time with the aim of imaging, respiratory and oxygen dynamics monitoring, blood gas examination, the function of circulatory system and the condition of other organs [2]. Blood oxygen saturation, blood gas, hemodynamics and respiratory mechanics are still routine and convenient monitoring methods of mechanical ventilation. Traditional lung images, such as X-ray, CT, MRI, certainly have the characteristics of clear images and easy analysis and diagnosis, but they are complicated to operate under the special circumstances of isolation and transportation of patients infected with new coronavirus. The chest electrical impedance tomography cannot provide clear image, but it is convenient to operate and can be continuously imaged [5]. Ultrasound lung images also have unique advantages in the diagnosis of pneumonia and the effect of ventilation [6]. These two methods can be real-time bedside monitoring, which are simple and practical to guide lung recruitment, to diagnose pneumonia, and to evaluate the mechanical ventilation effectiveness. In addition, while monitoring respiratory mechanics and oxygenation parameters during mechanical ventilation, we should pay close attention to the corresponding changes in the circulatory system and make timely adjustments.

\section{Electrical Impedance Tomography}

Electrical Impedance Tomography (EIT) is to use the impedance changes of living organisms or biological tissues, biological organs, and biological cells under the action of a safe current below the excitability threshold to obtain the organism internal resistance rate of distribution and changing images through image reconstruction $[5,7]$. The resistivity of different tissues or the same tissue under different physiological and pathological conditions is different. The periodic changes of air and blood flow in the lungs together determine the changes in the electrical impedance of the chest. The advantage of EIT lies in the use of the rich physiological and pathological information carried by bio-impedance to obtain damage-free functional imaging and medical image monitoring. Chest X-rays and CT are widely used in the diagnosis of lung infections. But they cannot monitor lung lesions in real time, cannot measure lung ventilation status, and most importantly cannot be used in patients with severe pneumonia and respiratory failure who cannot easily access these examination, so their application are limited. Lung EIT, as a brand new medical imaging technology, which is different from traditional imaging technology and conventional lung function monitoring, has outstanding features such as injury-free, portable, low-cost, functional imaging, and image monitoring. EIT can real-time dynamic monitor the pulmonary ventilation and blood flow distribution, evaluate the effectiveness of clinical treatment methods such as mechanical ventilation by measuring electrical resistance under different ventilation conditions [5,7].

At present, the commonly used methods to monitor the effectiveness of lung recruitment strategy and the suitability of PEEP include arterial blood gas analysis, peripheral oxygen saturation, pulmonary and chest maximum compliance, static pressure volume curve and so on, but these methods cannot meet the requirements of dynamic monitoring of regional lung perfusion. A number of studies have showed that in mechanical ventilation patients with ARDS, EIT has been used to accurately measure the whole lung and regional lung ventilation distribution, to show the influence of PEEP changes on alveolar expansion and collapse by gradually increasing and decreasing PEEP level, and in the end to obtain the optimal value of PEEP, which improves the ratio of ventilation and blood flow $(\mathrm{V} / \mathrm{Q})$, and plays an important role in individulized lung protective ventilation strategy $[5,7]$.

\section{Pulmonary Ultrasound}

Bedside lung ultrasound can be used for the diagnosis and differential diagnosis of various lung diseases by using a lowfrequency convex probe of 3 to $5 \mathrm{MHz}$ and a high-frequency linear probe of 8 to $12 \mathrm{MHz}$ [8]. Normal lung ultrasound images include bat sign, lung sliding sign, and A-line. Pathological images mainly include abnormal pleural lines, pulmonary consolidation, interstitial syndrome, fragmentation sign, dynamic bronchial signs, pleural effusion and soon [9].

With the development of ultrasound technology, pulmonary ultrasound is gradually found to be of great value in diagnosing acute respiratory distress syndrome, pulmonary edema, pneumonia, pneumothorax, pulmonary embolism and so on $[6,10,11]$. It can be used to monitor the changes in lung ventilation, to guide clinical fluid management and evaluate prognosis, especially in patients with severe diseases. Since chest X-rays and CT examinations are unsuitable for rapid diagnosis of critical diseases due to the shortages 
of inconvenient carrying, radiation exposition, poor reproducibility, position limitations, and high costs, and compared with chest CT, bedside lung ultrasound has advantages of non-invasive, dynamic and repeatable observation of patients with lung disease.

\section{The Advantage of Drainage Position Ventilation}

At present, prone position mechanical ventilation is widely used in patients infected with new coronavirus, which may be helpful to the drainage of pulmonary inflammation and the reduction of pulmonary shunt volume. 2 So far, no effective antiviral drugs have been found in defeating new coronavirus, so drainage becomes an important treatment for pulmonary inflammatory lesions. Because of inflammatory lesions in different parts of the lung, prone position ventilation is not suitable for all patients, and it may be more beneficial to adopt drainage position mechanical ventilation combined with tracheal suction with the infected side of lung lesions upper side. For example, the lateral and head-down position mechanical ventilation with the inflammatory lung upper side according to the characteristics of pulmonary imaging of some patients infected with new coronavirus. The lateral prone position can be tried to improve the inflammatory side lung ventilation, reduce pulmonary shunt, increase blood reflux and improve hemodynamics. However, it is important to avoid excessive head down, which increases abdominal pressure on the chest cavity.

In summary, based on the autopsy, clinical manifestations, lung pathological characteristics and present treatment of the patients infected with the new coronavirus, this article describes some possible improvement measures for the mechanical ventilation strategy. We believe that postural drainage ventilation, real-time bedside pulmonary ultrasound and chest electrical impedance monitoring will improve the clinical treatment of critical patients based on the previous guidelines for ARDS treatment. These methods provide some new ideas for clinical treatment and need to be used and verified in future clinical work.

\section{References}

1. Guan WJ, Ni ZY, Hu Y, Liang WH, Ou CQ, et al. (2020) Clinical characteristics of coronavirus disease 2019 in China. $N$ Engl J Med.

2. Lingzhong Meng, Haibo Qiu, Li Wan, Yuhang Ai, Zhanggang Xue, et al. (2020) Intubation and Ventilation amid the COVID-19 Outbreak: Wuhan's Experience. Anesthesiology 132: 1317-1332. [crossref]

3. Huang C, Wang Y, Li X, Ren L, Zhao J, et al. (2020) Clinical features of patients infected with 2019 novel coronavirus in Wuhan, China. Lancet 395: 497-506.

4. Qin Liu, Rongshuai wang, Guoqiang Qu, Yunyun wang, Pan Liu, et al. (2020) Gross Observation Report on the Autopsy of a nCov-2019 Pneumonia Death. Journal of Forensic Medicine (Chinese) 36: 21-23. [crossref]

5. Hsu CF, Cheng JS, Lin WC, Cheng KS, Lin SH, et al. (2016) Electrical impedance tomography monitoring in acute respiratory distress syndrome patients with mechanical ventilation during prolonged positive end-expiratory pressure adjustments [J]. J Formos Med Assoc 115: 195-202. [crossref]

6. Staub LJ, Mazzali Biscaro RR, Kaszubowski E, Maurici R (2019) Lung ultrasound for the emergency diagnosis of pneumonia, acute heart failure, and exacerbations of chronic obstructive pulmonary disease / asthma in adults: a systematic review and meta-analysis. J Emerg Med 56: 53-69. [crossref]

7. Heines SJH, Strauch U, Van de Poll MCG, Paul MHJR, Dennis CJJB (2018) Clinical implementation of electric impedance tomography in the treatment of ARDS: a single centre experience [J]. J Clin Monit Comput. [crossref]

8. Rouby JJ, Arbelot C, Gao YZ, Zhang M, Lv J, et al. (2018) APECHO Study Group. Training for lung ultrasound score measurement in critically ill patients. Am J Respir Crit Care Med 198: 398-401. [crossref]

9. Lichtenstein DA (2015) BLUE-protocol and FALLS-protocol: two applications of lung ultrasound in the critically ill. Chest 147: 1659-1670.

10. Chavez MA, Shams N, Ellington LE, Naithani N, Gilman RH, et al. (2014) Lung ultrasound for the diagnosis of pneumonia in adults: a systematic review and metaanalysis. Respir Res 15: 50. [crossref]

11. Long L, Zhao HT, Zhang ZY, Wang GY, Zhao HL (2017) Lung ultrasound for the diagnosis of pneumonia in adults: a meta-analysis. Medicine (Baltimore) 96: e5713. [crossref]

\section{Citation:}

Zhongping Cao (2020) Application of Drainage Position Ventilation and Real-Time Bedside Monitoring in Mechanical Ventilation of Patients Infected with nCov-19. Internal Med Res Open J Volume 5(4): 1-3. 